\title{
Magnetocrystalline anisotropy imprinting of an antiferromagnet on an amorphous ferromagnet in $\mathrm{FeRh} / \mathrm{CoFeB}$ heterostructures
}

Yali Xie (1)', Qingfeng Zhan $\mathbb{E}^{2}$, Yong $\mathrm{Hu}^{3,4}$, Xiao Hu${ }^{2}$, Xiaodan $\mathrm{Chi}^{3}$, Chenyu Zhang ${ }^{3}$, Huali Yang ${ }^{1}$, Wenhui Xie ${ }^{2}$, Xiaoyan Zhu', Jianhua Gao ${ }^{5}$, Wenjuan Cheng ${ }^{2}$, Dongmei Jiang ${ }^{2}$ and Run-Wei Li (i)

\begin{abstract}
Magnetic anisotropy is a fundamental key parameter of magnetic materials that determines their applications. For ferromagnetic materials, the magnetic anisotropy can be easily detected by using conventional magnetic characterization techniques. However, due to the magnetic compensated structure in antiferromagnetic materials, synchrotron measurements, such as X-ray magnetic linear dichroism, are often needed to probe their magnetic properties. In this work, we observed an imprinted fourfold magnetic anisotropy in the amorphous ferromagnetic layer of FeRh/CoFeB heterostructures. The MOKE and ferromagnetic resonance measurements show that the easy

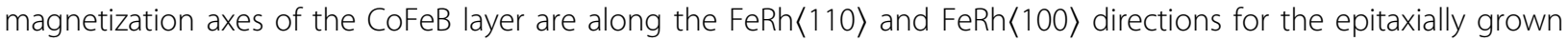
FeRh layer in the antiferromagnetic and ferromagnetic states, respectively. The combined Monte Carlo simulation and first-principles calculation indicate that the fourfold magnetic anisotropy of the amorphous CoFeB layer is imprinted due to the interfacial exchange coupling between the CoFeB and FeRh moments from the magnetocrystalline anisotropy of the epitaxial FeRh layer. This observation of imprinting the magnetocrystalline anisotropy of antiferromagnetic materials on easily detected ferromagnetic materials may be applied to probe the magnetic structures of antiferromagnetic materials without using synchrotron methods.
\end{abstract}

\section{Introduction}

Magnetic anisotropy is a fundamental key parameter of magnetic materials, which determines the application of various magnetic materials ${ }^{1}$. A permanent magnet usually possesses a high magnetic anisotropy to improve both the magnetic coercivity and the magnetic energy product. In contrast, a soft magnetic material needs a magnetic anisotropy as weak as possible to reduce the hysteresis loss

Correspondence: Qingfeng Zhan (qfzhan@phy.ecnu.edu.cn) or Run-Wei Li (runweili@nimte.ac.cn)

${ }^{1}$ Key Laboratory of Magnetic Materials and Devices, Ningbo Institute of Material Technology and Engineering, Chinese Academy of Sciences, Ningbo 315201, People's Republic of China

${ }^{2}$ Key Laboratory of Polar Materials and Devices (MOE) and State Key Laboratory of Precision Spectroscopy, School of Physics and Electronic Science, East China Normal University, Shanghai 200241, People's Republic of China Full list of author information is available at the end of the article These authors contributed equally: Yali Xie, Qingfeng Zhan and obtain a high permeability. Generally, the magnetic anisotropy of magnetic materials contains both intrinsic and extrinsic parts. The intrinsic magnetocrystalline anisotropy originates from the anisotropy of spin-orbit coupling ${ }^{2,3}$. Thus, the symmetry of anisotropy is determined by the crystal structure of magnetic materials. For instance, a single crystal ferromagnetic (FM) material of Fe with a body-center cubic structure reveals cubic magnetocrystalline anisotropy. Correspondingly, an epitaxially grown Fe film displays a fourfold in-plane magnetic anisotropy, which results in the occurrence of unconventional multistepped magnetic transitions ${ }^{4,5}$. Similarly, a single crystal anti-FM (AF) material also displays a magnetocrystalline anisotropy. However, due to the magnetic compensated structure, synchrotron measurements, such as X-ray magnetic linear dichroism, are often needed to probe its magnetic properties ${ }^{6}$. In

\section{(c) The Author(s) 2020}

(c) Open Access This article is licensed under a Creative Commons Attribution 4.0 International License, which permits use, sharing, adaptation, distribution and reproduction cc) in any medium or format, as long as you give appropriate credit to the original author(s) and the source, provide a link to the Creative Commons license, and indicate if changes were made. The images or other third party material in this article are included in the article's Creative Commons license, unless indicated otherwise in a credit line to the material. If material is not included in the article's Creative Commons license and your intended use is not permitted by statutory regulation or exceeds the permitted use, you will need to obtain permission directly from the copyright holder. To view a copy of this license, visit http://creativecommons.org/licenses/by/4.0/. 
contrast, amorphous magnetic materials do not display magnetocrystalline anisotropy because of the absence of long-range order in the crystal lattice, which makes them good candidates for soft magnetic materials. Extrinsic magnetic anisotropy is usually induced by the shape of the magnetic materials, the applied stress, the processing techniques, and the interfacial exchange coupling ${ }^{7}$. The exchange bias (EB) anisotropy is one of the most important extrinsic magnetic anisotropies and was discovered 60 years ago by Meiklejohn and Bean in a composite system containing an FM material coupled with the adjacent $\mathrm{AF}$ material ${ }^{8}$. In recent years, this effect has been employed to stabilize the magnetization in spintronic devices ${ }^{9,10}$, enhance the FMR frequency in microwave devices ${ }^{11}$, and induce magnetoelectric coupling in multiferroic composites ${ }^{12}$.

CoFeB alloys show excellent soft magnetic behaviors because of the absence of magnetocrystalline anisotropy caused by the amorphous crystal structure. The additional behavior of high spin polarization makes $\mathrm{CoFeB}$ extensively applied in multilayered spintronic devices ${ }^{13,14}$. On the other hand, the CsCl-type FeRh alloy is an important metamagnetic material. This material is $\mathrm{AF}$ at room temperature and undergoes a peculiar first-order phase transition to the FM phase with a low magnetocrystalline anisotropy upon heating above a transition temperature $^{15,16}$. Most previous research has been focused on the magnetic and transport behaviors of FeRh caused by the phase transition, such as the magnetic entropy change ${ }^{17}$, the magnetoresistance effect ${ }^{18}$, the reduction in resistivity $^{19}$, and the nucleation of magnetic domains ${ }^{20}$. Recently, the interfacial exchange coupling between thin-film FeRh and various FM layers has attracted extensive attention due to its potential application in magnetic recording and spin-valve sensors. Suzuki et al. observed an EB in Fe/ $\mathrm{FeRh}(111)$ bilayers in the AF state of FeRh but no occurrence of an $\mathrm{EB}$ at the $\mathrm{Fe} / \mathrm{FeRh}(001)$ interface ${ }^{21}$. Nam et al. reported a very large EB anisotropy up to $0.8 \mathrm{erg} / \mathrm{cm}^{2}$ in $\mathrm{FePt} / \mathrm{FeRh}(001)$ bilayers $^{22}$. Above the FeRh transition temperature, $\mathrm{FePt} / \mathrm{FeRh}(001)$ forms an exchange spring system, significantly lowering the coercive field of the composite system, which opens possible media applications for thermally assisted magnetic recording ${ }^{23}$. In this work, we investigated the magnetic behaviors of magnetic heterostructures with an amorphous $\mathrm{CoFeB}$ layer grown on an epitaxial FeRh layer. It is of great interest that the amorphous $\mathrm{CoFeB}$ layer displays an in-plane fourfold magnetic anisotropy, which is usually observed in single crystal films. The MOKE and FMR measurements show that the easy axes of the CoFeB layer are along the FeRh $\langle 110\rangle$ and $\mathrm{FeRh}\langle 100\rangle$ directions for the FeRh layer in the AF and FM states, respectively. The combined Monte Carlo simulation and first-principles calculation indicate that the fourfold magnetic anisotropy of the amorphous
CoFeB layer is imprinted due to the interfacial exchange coupling between the $\mathrm{CoFeB}$ and FeRh moments from the magnetocrystalline anisotropy of the epitaxial FeRh layer regardless of the magnetic states of FeRh. This phenomenon may be applied to probe the magnetic structures of AF materials without using synchrotron methods.

\section{Materials and methods \\ Sample preparation}

$\mathrm{Co}_{40} \mathrm{Fe}_{40} \mathrm{~B}_{20}(\mathrm{CoFeB}) / \mathrm{FeRh}$ bilayers with an FeRh thickness fixed at $30 \mathrm{~nm}$ and a CoFeB thickness $t_{\mathrm{CoFeB}}$ varied from 5 to $30 \mathrm{~nm}$ were deposited on commercial (001)oriented $\mathrm{MgO}$ substrates in a magnetron sputtering system with a base pressure below $1.0 \times 10^{-8}$ Torr. Before deposition, the substrates were annealed at $500^{\circ} \mathrm{C}$ for $1 \mathrm{~h}$ in a vacuum chamber. FeRh layers were grown at a temperature of $530^{\circ} \mathrm{C}$ and then annealed at $650{ }^{\circ} \mathrm{C}$ for $1 \mathrm{~h}$. After the samples were cooled to room temperature, the $\mathrm{CoFeB}$ layers were deposited on top of the FeRh layers. Prior to removal from the vacuum chamber, the samples were capped by a $3 \mathrm{~nm}$ Ta layer to avoid oxidation. The film thicknesses were controlled by the deposition time, which was calibrated by X-ray reflectivity.

\section{Characterization methods}

The epitaxial growth of FeRh films was characterized by in-plane X-ray $\phi$-scans. The microstructure of the CoFeB/ FeRh bilayers was observed by a high-resolution transmission electron microscope (HRTEM). The magnetic switching processes were measured by using a combined longitudinal $(||)$ and transverse $(\perp)$ magneto-optical Kerr effect (MOKE) setup. The temperature-dependent magnetic behaviors were characterized by using a magnetic property measurement system (MPMS, Quantum Design, USA). The magnetic anisotropy was quantitatively measured by FM resonance (FMR) spectroscopy at a radio frequency of $9.31 \mathrm{GHz}$.

\section{Monte Carlo simulations}

Monte Carlo simulations were performed using a technique based on a simulated annealing algorithm ${ }^{24}$. A soft magnet (SM) system was first zero-field cooled from an initial temperature $T_{0}=1.0 J_{\mathrm{SM}} / k_{\mathrm{B}}$ at a constant step of $-0.05 J_{\mathrm{SM}} / k_{\mathrm{B}}$ to a target temperature $T=0.05 J_{\mathrm{SM}} / k_{\mathrm{B}}$, which is much lower than the magnetic ordering temperature, where $J_{S M}$ is the exchange constant of the SM to serve as an energy unit and $k_{\mathrm{B}}$ is the Boltzmann constant. Then, the magnetization hysteresis loop is recorded by isothermal cycling of $H$ between $1.0 J_{\mathrm{SM}} / g \mu_{\mathrm{B}}$ and $-1.0 J_{\mathrm{SM}} /$ $g \mu_{\mathrm{B}}$ at constant steps of $\left|0.02 J_{\mathrm{SM}} / g \mu_{\mathrm{B}}\right|$, where $g$ is the Landé $g$-factor and $\mu_{\mathrm{B}}$ is the Bohr magneton. At each temperature/field value, $10^{5}$ Monte Carlo steps were used to equilibrate the system and then discarded, followed by $10^{5}$ Monte Carlo steps for averaging magnetization 
quantities ${ }^{25,26}$. Finally, 50 sets of independent initial spin states were chosen to minimize the errors.

\section{First-principles calculation}

The first-principles spin-polarized calculations were carried out by using the Vienna ab initio simulation package with the projector augmented wave $\operatorname{method}^{27,28}$. The exchange-correlation interactions were treated via generalized gradient approximations with the Perdew-Burke-Ernzerhof functional ${ }^{29}$. The plane-wave cutoff energy was $400 \mathrm{eV}$. Monkhorst-Pack meshes of $10 \times 10 \times 10$ were employed for geometry optimization. The magnetocrystalline anisotropy energy (MAE) was calculated by performing non-collinear spin-polarized calculations $^{30}$. To obtain reliable values for the MAEs, the convergence accuracy of the energy was $10^{-7} \mathrm{eV}$, and dense $k$ points of $20 \times 20 \times 20$ were used for the calculations. The MAEs for FeRh in different spin arrangements were calculated in a non-self-consistent way by using the charge density and wavefunctions of a collinear spinpolarized calculation. The magnetic anisotropy was determined when the orientations of the magnetic moment were set along the FeRh $\langle 100\rangle$ and FeRh $\langle 110\rangle$ axes.

\section{Results and discussion}

Figure 1a shows the temperature-dependent magnetization curve measured with an in-plane magnetic field of $2 \mathrm{kOe}$ for a $30 \mathrm{~nm}$ FeRh film epitaxially grown on $\mathrm{MgO}$ (001). The magnetization of the FeRh film is as small as $21 \mathrm{emu} / \mathrm{cm}^{3}$ at room temperature and then increases to $1220 \mathrm{emu} / \mathrm{cm}^{3}$ at $380 \mathrm{~K}$ during the heating process, indicating a typical AF-FM phase transition of FeRh. In the cooling process, the magnetization of the FeRh film gradually decreases to the initial value with a remarkable temperature hysteresis, indicating the first-order phase transition of FeRh. The hysteresis loops measured at $400 \mathrm{~K}$, as shown in the inset of Fig. 1a, indicate that the inplane easy and hard magnetization axes are along the FeRh[100] and FeRh[110] directions, respectively. After growing the $\mathrm{CoFeB}$ layer on top of the FeRh layer, the magnetization of a typical sample of $\mathrm{CoFeB}(15 \mathrm{~nm}) / \mathrm{FeRh}$ $(30 \mathrm{~nm})$ increases from $780 \mathrm{emu} / \mathrm{cm}^{3}$ at room temperature to $1930 \mathrm{emu} / \mathrm{cm}^{3}$ at $380 \mathrm{~K}$ during the heating process, as shown in Fig. S1b of the Supporting Information. The corresponding critical phase transition temperatures of the FeRh film determined from the differential temperature-dependent magnetization curve are 360 and $345 \mathrm{~K}$ in the heating and cooling processes, respectively (Supporting Information, Fig. S1c). The X-ray $\phi$-scans of the $\mathrm{CoFeB}(15 \mathrm{~nm}) / \mathrm{FeRh}(30 \mathrm{~nm})$ bilayer with a fixed $2 \theta$ at the $\mathrm{FeRh}(011)$ reflection display a $90^{\circ}$ interval of four peaks. The FeRh peaks are located at $45^{\circ}$ with respect to the $\mathrm{MgO}$ peaks, as shown in Fig. 1b. These features indicate that the FeRh films are epitaxially grown with a $45^{\circ}$ in-plane structure rotation on the $\mathrm{MgO}(001)$ substrates, that is, the epitaxial relationships of $\mathrm{FeRh}(001)$ $\langle 110\rangle / / \mathrm{MgO}(001)\langle 100\rangle$. No X-ray diffraction peak from $\mathrm{CoFeB}$ is detected, indicating an amorphous structure of the $\mathrm{CoFeB}$ layers. A clear interface between the $\mathrm{CoFeB}$ and FeRh layers can be observed in the cross-sectional HRTEM image, as shown in Fig. 1c. Most of the CoFeB region looks amorphous, and the corresponding fast Fourier transform (FFT) image displays an intense diffusive ring feature. However, a strip-like contrast can be seen in the CoFeB region within $0.5-1.0 \mathrm{~nm}$ from the FeRh interface, which indicates the partial crystallization of $\mathrm{CoFeB}$ near the interface. The lattice fringe spacings of $0.297 \pm 0.002, \quad 0.211 \pm 0.001, \quad$ and $\quad 0.172 \pm 0.001 \mathrm{~nm}$ obtained in the FeRh region agree well with those of the (001), (110), and (111) planes of FeRh, respectively. The corresponding FFT image shows clear diffraction spots. In addition, the HRTEM image of a reference $\mathrm{CoFeB}(15 \mathrm{~nm})$ film fabricated on $\mathrm{MgO}(001)$ substrate at room temperature indicates the similar partial crystallization of $\mathrm{CoFeB}$ within $\sim 1.3 \mathrm{~nm}$ from the $\mathrm{CoFeB} / \mathrm{MgO}$ interface, as shown in Fig. S2 of the Supporting Information.

Both longitudinal $(\|)$ and transverse $(\perp)$ MOKE loops were obtained at various in-plane magnetic field orientations $\phi$ with respect to the $\mathrm{MgO}[100]$ direction, i.e., the $\mathrm{FeRh}[1 \overline{1} 0]$ direction, as summarized in Fig. 1d. Before the MOKE measurements, to induce an $\mathrm{EB}$, the samples were heated beyond the AF-FM transition temperature to $400 \mathrm{~K}$ and then cooled down to $300 \mathrm{~K}$ with an external magnetic field of $2 \mathrm{~T}$ along the $\mathrm{MgO}[100]$ direction in MPMS. Four kinds of MOKE loops are observed for the typical $\mathrm{CoFeB}(15 \mathrm{~nm}) / \mathrm{FeRh}(30 \mathrm{~nm})$ bilayer measured at different $\phi$ at room temperature, as depicted in Fig. 2. The spin orientations of $\mathrm{CoFeB}$ in each step are also presented according to the transverse MOKE signal. Notably, since the penetration depth of the laser used in our MOKE setup is known to be approximately $10 \mathrm{~nm}$ and the Ta capping layer is $3 \mathrm{~nm}$ in thickness ${ }^{31}$, the MOKE signal is detected by illuminating the samples from the top side in our measurements, which comes mostly from the amorphous $\mathrm{CoFeB}$ but not from the partially crystallized $\mathrm{CoFeB}$ near the interface and the below single crystal FeRh. A square loop with a coercivity $H_{\text {cI }}$ of $65 \mathrm{Oe}$ is observed for $\phi$ around the field-cooling (FC) direction, which is obviously larger than that $\left(H_{c}=15 \mathrm{Oe}\right.$, Supporting Information, Fig. S3) of a single-CoFeB layer without growing the $\mathrm{FeRh}$ underlayer, indicating the occurrence of interfacial exchange coupling between the $\mathrm{CoFeB}$ and $\mathrm{FeRh}$ moments. The corresponding transverse MOKE signal indicates that the $\mathrm{CoFeB}$ magnetization switches between the $\mathrm{MgO}[\overline{100}]$ and $\mathrm{MgO}[100]$ directions via domain wall (DW) nucleation and propagation (Fig. 2a). Only a very small loop-shift less than 3 Oe is 

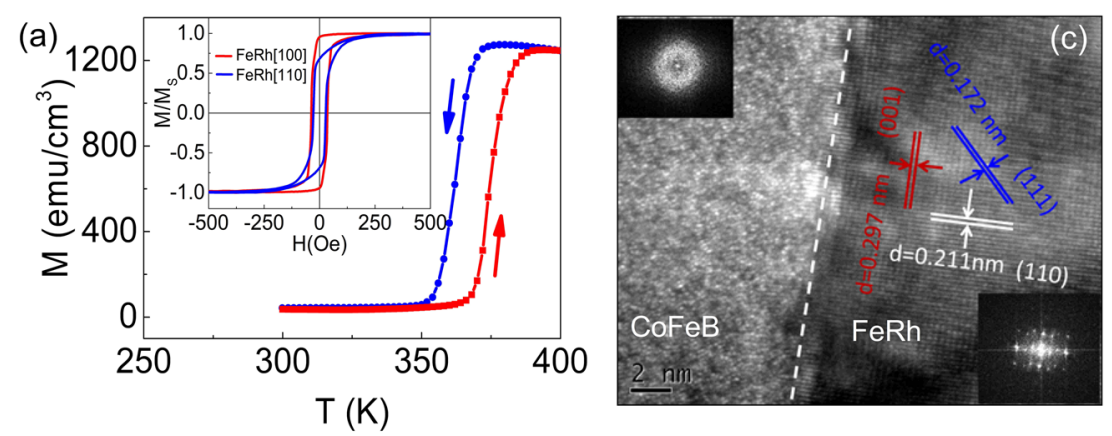

(b)
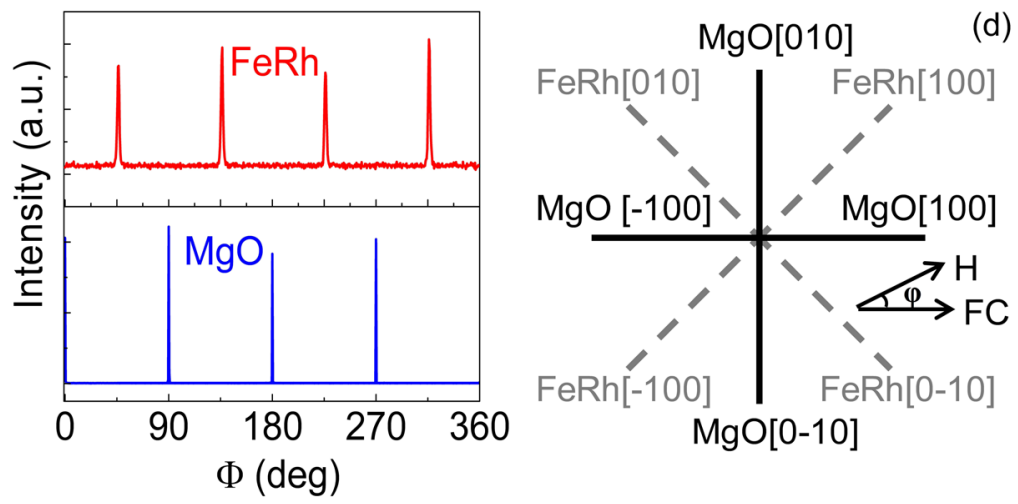

Fig. 1 Phase transition of FeRh and structural characterizations of CoFeB/FeRh/MgO. a Temperature-dependent magnetization curves of FeRh $(30 \mathrm{~nm}) / \mathrm{MgO}$. The inset shows the corresponding hysteresis loops measured along the FeRh[100] and FeRh[110] directions at 400K. $\mathbf{b}$ X-ray $\phi$-scans and $\mathbf{c}$ HRTEM and FFT images of CoFeB(15nm)/FeRh (30nm)/MgO. d The measurement and crystalline geometries for CoFeB/FeRh/MgO. The magnetic properties are obtained at various field orientations $(\varphi)$ with respect to the $\mathrm{MgO}[100]$ direction. Before the magnetic measurement, the samples are cooled down from 400 K at a magnetic field of 2T along the MgO[100] direction.

observed. Similar observations, i.e., only the enhancement of the coercive field but no obvious EB, have also been found in several similar bilayers, such as $\mathrm{Fe} / \mathrm{FeRh}^{21}$ and $\mathrm{NiFe} / \mathrm{FeRh}^{32}$. Asymmetrically shaped loops with a onestep magnetic transition for the descending magnetic field branch but a two-step magnetic transition for the ascending magnetic field branch are observed near $\phi=$ $20^{\circ}$ (Fig. 2b). The lower and higher coercive fields for a branch of the hysteresis loop are defined as $H_{\mathrm{cI}}$ and $H_{\mathrm{cII}}$, respectively. This kind of asymmetrically shaped loop has been reported in several epitaxial EB bilayers, including $\mathrm{Fe} / \mathrm{MnF}_{2}{ }^{33}$ and $\mathrm{Fe} / \mathrm{MnPd}^{34}$. The observation of an asymmetrically shaped loop suggests that the EB is likely established in $\mathrm{CoFeB} / \mathrm{FeRh}$ bilayers. When the field orientation is farther away from the $\mathrm{MgO}[100]$ direction, for instance, $\phi=40^{\circ}$, one can observe two-step hysteresis loops. The transverse MOKE loops show that in the intermediate states for the ascending and descending field branches, $\mathrm{CoFeB}$ spins lie on the two opposite directions oriented perpendicular to the initial and final remnant axes (Fig. 2c), indicating that the magnetic switching processes are mediated by two separated $90^{\circ} \mathrm{DW}$ nucleations. This kind of multistepped magnetic switching process has been observed for many FM films with cubic crystal structures that have an intrinsic cubic magnetocrystalline anisotropy superimposed with an extra uniaxial magnetic anisotropy and/or a unidirectional anisotropy ${ }^{4,5,35}$. When the field orientation is perpendicular to the FC direction, that is, $\phi=90^{\circ}$, another kind of two-step loop in which the magnetic switching routes for both branches are mediated via the FC direction can be observed (Fig. 2d). The occurrence of this one-sided twostep loop confirms that a unidirectional EB anisotropy is established in the FC process $^{35}$.

When the temperature increases to $400 \mathrm{~K}$, where the AF-FM phase transition of FeRh is completely finished, asymmetric and one-sided two-step magnetic switching processes are no longer observed (Supporting Information, Fig. S4), indicating the disappearance of the EB. The hysteresis loop with a coercivity $H_{\mathrm{cI}}=26$ Oe at $\phi=0^{\circ}$ is still obviously larger than that of a single $\mathrm{CoFeB}$ layer without growing the $\mathrm{FeRh}$ underlayer, indicating the occurrence of interfacial exchange spring coupling between $\mathrm{CoFeB}$ and $\mathrm{FeRh}$. Although the longitudinal MOKE signals show a single hysteresis loop behavior at different $\phi$ values, the corresponding transverse signal indicates that the magnetic switching processes are actually two-sided two-step processes with two coercivities without an EB. The easy magnetization axis of $\mathrm{CoFeB}$ is along the $\mathrm{MgO}[100]$ direction, i.e., the $\mathrm{FeRh}[110]$ 

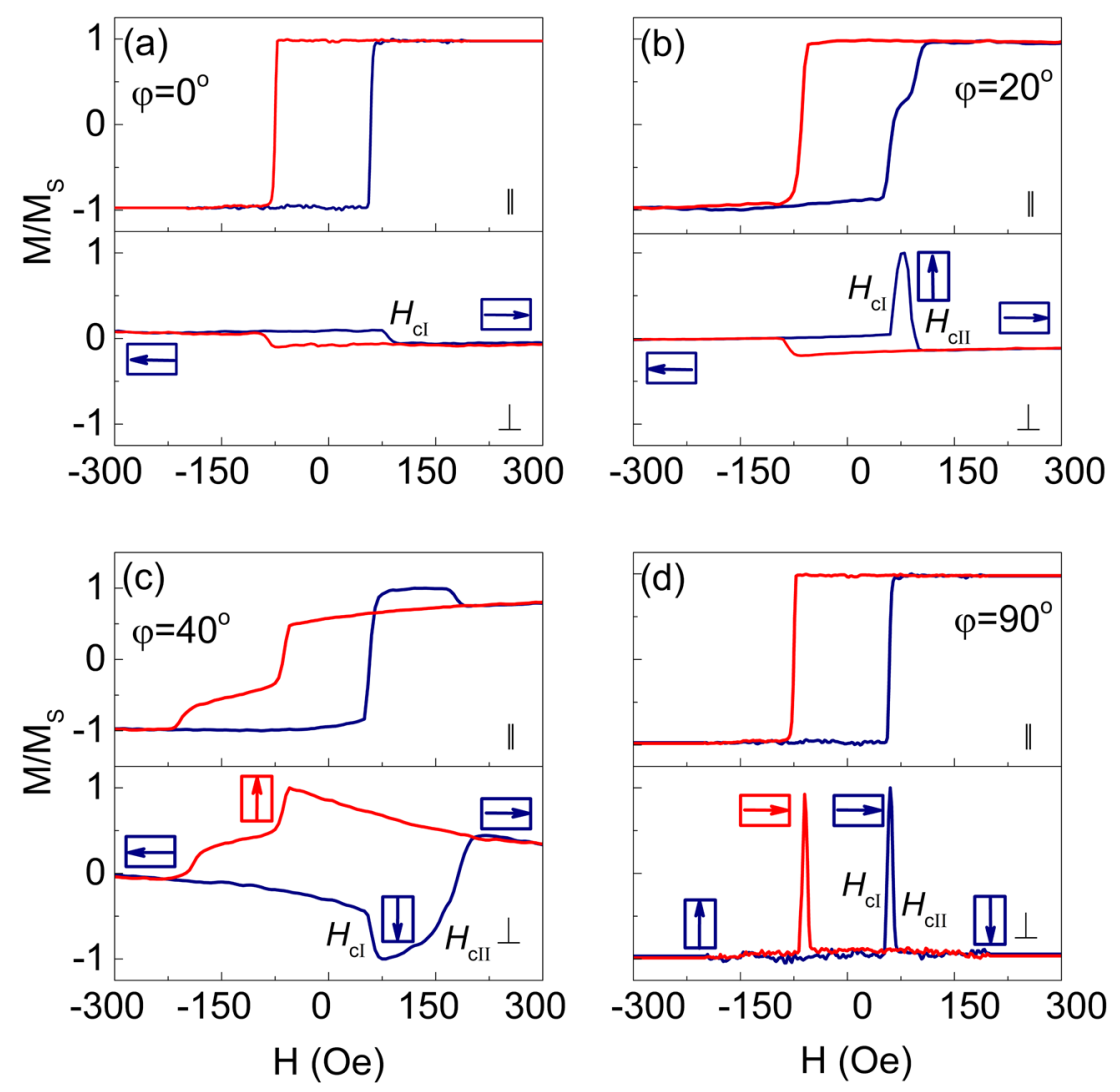

Fig. 2 MOKE loops measured at various field orientations. Longitudinal (II) and transverse $(\perp)$ MOKE loops for the CoFeB(15nm)/FeRh(30nm) film measured at $\mathbf{a} \varphi=0^{\circ}, \mathbf{b} \varphi=20^{\circ}, \mathbf{c} \varphi=40^{\circ}$, and $\mathbf{d} \varphi=90^{\circ}$ at room temperature. The blue (red) curves are for applied fields varying from negative (positive) to positive (negative) saturation. The orientations of CoFeB spins in the magnetic switching processes are represented by the arrows enclosed in a square. The coercive fields are also indicated in the hysteresis loops.

direction, for FeRh in the AF state at room temperature but then develops along the $\mathrm{MgO}[110]$ direction, i.e., the $\mathrm{FeRh}[100]$ direction, for FeRh in the FM state at $400 \mathrm{~K}$, as shown in Fig. S5 of the Supporting Information. These magnetic behaviors can also be observed in the other samples with $t_{\mathrm{CoFeB}}=5,10$, and $30 \mathrm{~nm}$.

Figure $3 \mathrm{a}$ presents the magnetic field orientation dependence of the switching fields for the ascending field branch in the $\mathrm{CoFeB}(15 \mathrm{~nm}) / \mathrm{FeRh}(30 \mathrm{~nm})$ bilayer measured at room temperature, which reveals an obvious fourfold symmetry about the in-plane orthogonal $\mathrm{MgO}$ [100] and $\mathrm{MgO}[010]$ directions. Because the $\mathrm{EB}$ of $\mathrm{CoFeB} /$ FeRh bilayers is negligible, the magnetic switching fields for the descending field branch are almost identical to that of the ascending field branch except the asymmetrically shaped loops appearing around $\phi=20^{\circ}$. In contrast, a single $\mathrm{CoFeB}$ layer displays only a weak uniaxial magnetic anisotropy, as shown in Fig. S3 of the Supporting Information. Previously, this kind of angular dependent magnetic behavior can only be observed in epitaxially grown FM films or EB bilayers in which the intrinsic fourfold magnetocrystalline anisotropy is superimposed by an extra uniaxial magnetic anisotropy and/or a unidirectional magnetic anisotropy ${ }^{4,5,35}$. Although it is impossible for a single $\mathrm{CoFeB}$ layer to possess an intrinsic fourfold magnetocrystalline anisotropy due to the amorphous crystal structure, we are hesitant to fit such angular dependent behaviors by using a phenomenological model based on $90^{\circ} \mathrm{DW}$ nucleation and propagation in a magnetic system with a fourfold magnetic anisotropy $K_{1}$ along the $\mathrm{MgO}$ $\langle 100\rangle$ directions and a uniaxial magnetic anisotropy $K_{u}$ along the $\mathrm{MgO}[100]$ direction. The unidirectional magnetic anisotropy $K_{e b}$ is not considered due to the negligible strength. The corresponding theoretical switching fields for $90^{\circ}$ magnetic transitions are

$$
H_{c l, I I}=\frac{\varepsilon_{90^{\circ}} \pm K_{u}}{M( \pm \sin \phi \pm \cos \phi)},
$$

where $\varepsilon_{90^{\circ}}$ is the $90^{\circ} \mathrm{DW}$ nucleation energy, $M$ is the saturation magnetization, and the signs in the equation depend on the range of the magnetic field orientation $\phi$, 



Fig. 3 Angular dependence of the switching fields of CoFeB/FeRh. The experimentally measured magnetic switching fields $H_{c l}(\mathrm{dots})$ and $H_{c \mid l}$ (squares) as a function of the field orientation $\varphi$ and the corresponding theoretical curves for $H_{c 1}$ (red) and $H_{c 11}$ (blue) for CoFeB(15nm)/FeRh(30nm) bilayers $\mathbf{a}$ at room temperature and $\mathbf{c}$ at $400 \mathrm{~K}$. Dependence of the uniaxial magnetic anisotropy $K_{u}$ and the DW nucleation energies $\varepsilon_{90^{\circ}}$ and $\varepsilon_{90^{\circ}} \pm 2 \delta$ on the CoFeB thickness $t_{\mathrm{CoFeB}}$ obtained by fitting the angular dependent switching fields of CoFeB/FeRh bilayers to Eq. (1) $\mathbf{b}$ at room temperature and $\mathbf{d}$ at $400 \mathrm{~K}$. The curves indicate the corresponding theoretical fitting to $K_{u} / M=K_{u}^{S} / M t_{\mathrm{CoFeB}}, \varepsilon_{90^{\circ}} / M=\varepsilon_{90^{\circ}}^{S} / M t_{\mathrm{CoFeB}}$, and $\varepsilon_{90^{\circ} \pm 2 \delta} / M=\varepsilon_{90^{\circ} \pm 2 \delta}^{S} / M t_{\mathrm{CoFeB}}$.

as described elsewhere ${ }^{35-37}$. Figure $3 a$ indicates that the angular dependence of the magnetic switching fields for $\mathrm{CoFeB}(15 \mathrm{~nm}) / \mathrm{FeRh}(30 \mathrm{~nm})$ film can be nicely fitted, giving the parameters $\varepsilon_{90^{\circ}} / M=78 \pm 4 \mathrm{Oe}$ and $K_{u} / M=3 \pm 2$ Oe. The parameters $\varepsilon_{90^{\circ}} / M$ and $K_{u} / M$ for $\mathrm{CoFeB} / \mathrm{FeRh}$ bilayers turn out be inversely proportional to $t_{\mathrm{CoFeB}}$, as shown in Fig. 3b, by fitting to $\varepsilon_{90^{\circ}} / M=$ $\varepsilon_{90^{\circ}}^{S} / M t_{\mathrm{CoFeB}}$ and $K_{u} / M=K_{u}^{S} / M t_{\mathrm{CoFeB}}$, respectively, where $\varepsilon_{90^{\circ}}^{S} / M$ and $K_{u}^{S} / M$ are the interface-related coefficients, which suggests that the uniaxial magnetic anisotropy and the DW pinning are interface-related effects. Figure $3 \mathrm{c}$ shows the angular dependence of the magnetic switching fields for $\mathrm{CoFeB}(15 \mathrm{~nm}) / \mathrm{FeRh}(30 \mathrm{~nm})$ measured at $400 \mathrm{~K}$, which is similar to that of room temperature but with a phase shift of $45^{\circ}$. This indicates that the easy axes of the fourfold magnetic anisotropy are no longer along the $\mathrm{MgO}\langle 100\rangle / \mathrm{FeRh}\langle 110\rangle$ directions but along the $\mathrm{MgO}\langle 110\rangle / \mathrm{FeRh}\langle 100\rangle$ directions. Since the uniaxial magnetic anisotropy remains along the $\mathrm{MgO}$ [100] direction and is no longer collinear with the easy axis of the fourfold magnetic anisotropy, the magnetization does not switch exactly over $90^{\circ}$, but over $90^{\circ} \pm 2 \delta$, where $\delta$ is given by $\delta=\frac{1}{2} \sin ^{-1}\left(K_{u} / K_{1}\right)^{38}$. In our work, the value of $\delta$ is approximately $2^{\circ}$, as can be estimated from the following FMR measurements. The DW nucleation energies $\varepsilon_{90^{\circ} \pm 2 \delta}$ can be evaluated by using the theoretical switching fields in a revised form ${ }^{4}$, which results in the fitting parameters of $\varepsilon_{90^{\circ}-2 \delta} / M=26 \pm 1 \mathrm{Oe}$ and $\varepsilon_{90^{\circ}+2 \delta} / M=29 \pm 1$ Oe. These parameters also display an obvious $1 / t_{\mathrm{CoFeB}}$ dependence, as shown in Fig. $3 \mathrm{~d}$, by fitting to $\varepsilon_{90^{\circ} \pm 2 \delta} / M=\varepsilon_{90^{\circ} \pm 2 \delta}^{S} / M t_{\mathrm{CoFeB}}$, where $\varepsilon_{90^{\circ} \pm 2 \delta}^{S} / M$ is the interface-related coefficient.

The FMR measurements were employed to quantitatively evaluate the fourfold magnetic anisotropy and the uniaxial magnetic anisotropy of the CoFeB layer. Figure 4a shows the FMR derivative absorption spectra for $\mathrm{CoFeB}$ $(15 \mathrm{~nm}) / \mathrm{FeRh}(30 \mathrm{~nm})$ bilayers measured at different magnetic field orientations at room temperature. Only the $\mathrm{CoFeB}$ signal can be obviously detected in the magnetic field range from 700 to 3000 Oe at a radio frequency of 9.31 GHz, since FeRh is in the AF state at room temperature. The resonance field $H_{r}$ achieves four local minima at $\phi=0^{\circ}, 90^{\circ}, 180^{\circ}$, and $270^{\circ}$, indicating a fourfold magnetic anisotropy with easy axes along the $\mathrm{FeRh}\langle 110\rangle$ directions. The symmetry of the angular dependence of $H_{r}$ remains unchanged until the occurrence of the AF-FM phase transition of FeRh at $360 \mathrm{~K}$, as shown in Fig. 4b. For FeRh entering the FM state, the minima of $H_{r}$ change to appear at $\phi=45^{\circ}, 135^{\circ}, 225^{\circ}$, and $315^{\circ}$, indicating that the easy axes of the fourfold magnetic anisotropy of the $\mathrm{CoFeB}$ layer become along the $\mathrm{FeRh}\langle 100\rangle$ directions. It is noted that the FeRh film in the FM state displays a 

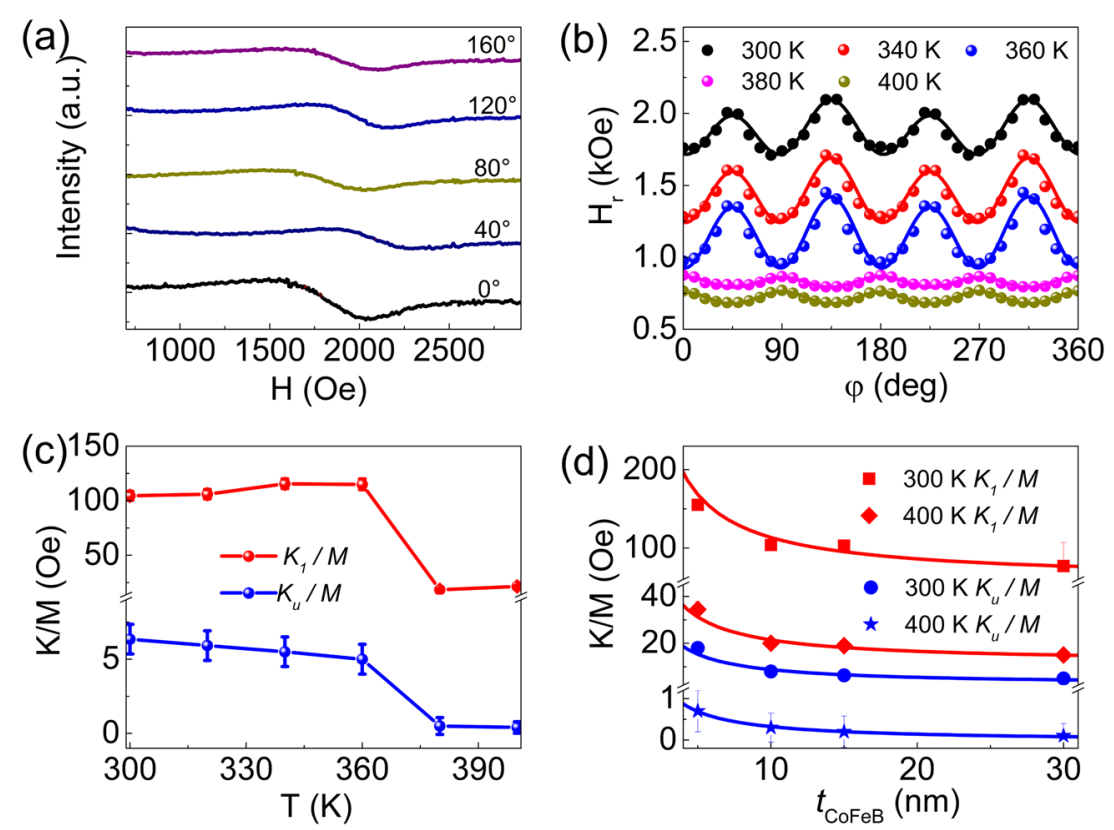

Fig. 4 Magnetic anisotropies of CoFeB/FeRh at different temperatures. a Typical FMR spectra for CoFeB(15nm)/FeRh (30nm) measured at different field orientations at 300K. b Typical angular dependence of the resonance field $H_{r}$ (dots) for CoFeB(15nm)/FeRh(30nm) measured at different temperatures and the corresponding theoretical results (curves) by fitting to Eq. (2). c Temperature dependence of the fourfold magnetic anisotropy $K_{1}$ and the uniaxial magnetic anisotropy $K_{u} \cdot \mathbf{d}$ CoFeB thickness $t_{\text {CoFeB }}$ dependence of $K_{1}$ and $K_{u}$ at 300 and $400 \mathrm{~K}$. Some associated error bars are too small to see. The curves indicate the corresponding theoretical fitting to $K_{1} / M=K_{1}^{S} / M t_{\text {CoFeB }}$ and $K_{u} / M=K_{u}^{S} / M t_{\text {CoFeB }}$.

resonance field below $600 \mathrm{Oe}$ at $9.4 \mathrm{GHz}^{39}$. The detected FMR signals above 700 Oe may come from the CoFeB layer. Obviously, a $45^{\circ}$ rotation of the fourfold magnetic anisotropy in the $\mathrm{CoFeB}$ layer takes place after the occurrence of the FeRh AF-FM phase transition, which is consistent with the MOKE loop measurements. The angular dependence of $H_{r}$ can be fitted by using a simplified equation derived from the Landau-Lifshitz equation by considering a fourfold magnetic anisotropy $K_{1}$ superimposed by a uniaxial magnetic anisotropy $K_{u}^{40}$ :

$$
\begin{aligned}
\left(\frac{\omega}{\gamma}\right)^{2}= & {\left[H_{r}+4 \pi M-\frac{3 K_{1}}{2 M}+\frac{2 K_{u}}{M} \cos 2 \phi+\frac{K_{1}}{2 M} \cos 4 \phi\right] } \\
& \times\left[H_{r}+\frac{4 K_{u}}{M} \cos 2 \phi+\frac{2 K_{1}}{M} \cos 4 \phi\right],
\end{aligned}
$$

where $\gamma$ is the gyromagnetic ratio. As a result, $K_{1} / M=$ $103 \pm 4$ Oe and $K_{u} / M=6 \pm 1$ Oe along the $\mathrm{MgO}[100]$ direction are obtained at $300 \mathrm{~K}$. After the occurrence of the AF-FM phase transition of FeRh, $K_{1} / M$ and $K_{u} / M$ (the easy axis keeps along the $\mathrm{MgO}[100]$ direction) steeply decrease to 18 and $0.3 \mathrm{Oe}$ at $380 \mathrm{~K}$, respectively (Fig. 4c). The fitting values of $K_{1} / M$ and $K_{u} / M$ measured at 300 and $400 \mathrm{~K}$ display an obvious $1 / t_{\mathrm{CoFeB}}$ dependence, as shown in Fig. 4 d, by fitting to $K_{1} / M=K_{1}^{S} / M t_{\mathrm{CoFeB}}$ and $K_{u} / M=K_{u}^{S} / M t_{\mathrm{CoFeB}}$, respectively, where $K_{1}^{S} / M$ and
$K_{u}^{S} / M$ are the interface-related coefficients. These features confirm that the fourfold magnetic anisotropy and the uniaxial magnetic anisotropy in the amorphous $\mathrm{CoFeB}$ layer are induced by an interfacial effect regardless of FeRh in the AF or FM states.

The magnetic behaviors of remarkably enhanced coercivity but very weak EB in $\mathrm{CoFeB} / \mathrm{FeRh}$ heterostructures can be understood by considering two kinds of FeRh moments at the interface. The rotatable and pinned uncompensated moments in the AF layer couple to the moments in the FM layers across the interface, giving rise to an enhancement of the coercivity and the EB in an FM/ AF bilayer, respectively ${ }^{41,42}$. The pinned uncompensated moments are tightly locked to the AF lattice and considered fixed under an external magnetic field. The rotatable uncompensated moments are strongly coupled to the FM moments and rotate with them during magnetization reversal. Brück et al. employed soft X-ray magnetic circular dichroism and magnetic reflectivity to quantitatively reveal that the pinned uncompensated $\mathrm{Mn}$ moments are one order of magnitude less than the rotatable Mn moments in MnPd/Fe EB heterostructures ${ }^{43}$. In addition, the uncompensated pinned Mn moments occur in a larger thickness than the rotatable Mn moments in an induced FM region at the interface. Similar observations have also been reported in $\mathrm{CoO} / \mathrm{Fe}$ bilayers ${ }^{44}$. In our $\mathrm{CoFeB} / \mathrm{FeRh}$ heterostructure, the clear enhancement of 
(a)

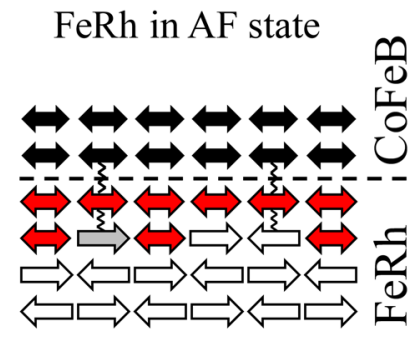

(c)



(b)

\section{FeRh in FM state}

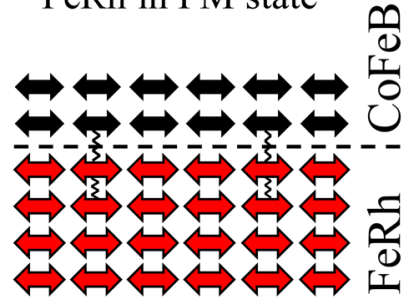

(d) (e)

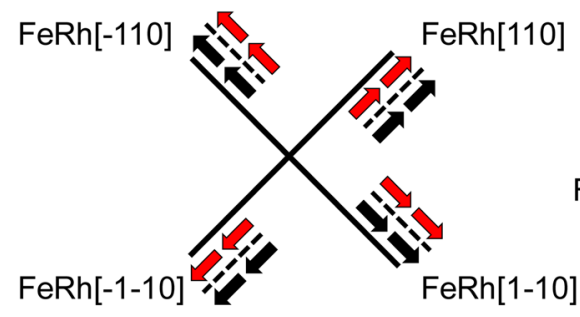

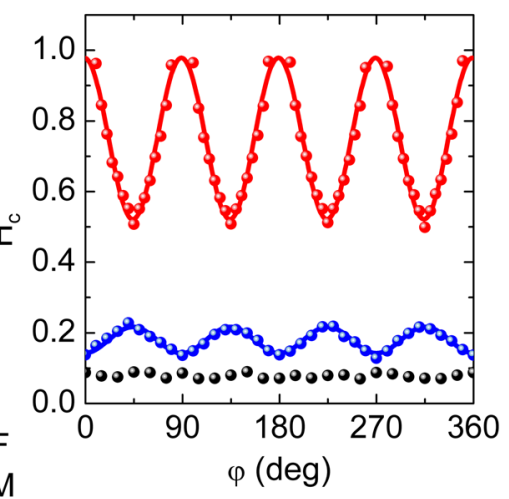

(f)

\section{FeRh[010]}

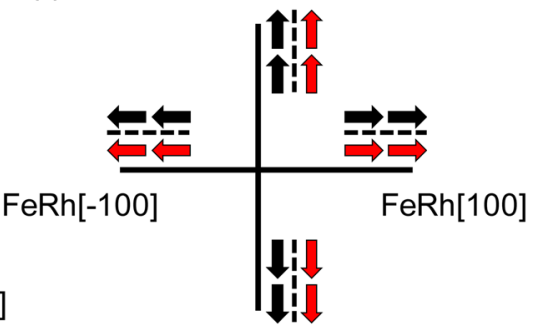

FeRh[0-10]

Fig. 5 Exchange coupling between CoFeB and FeRh moments across the interface. Sketches for the exchange coupling between CoFeB and FeRh moments across the interface for FeRh $\mathbf{a}$ in the AF state and $\mathbf{b}$ in the FM state. CoFeB moments and rotatable FeRh moments are represented by black and red double arrows, respectively. Uncompensated and compensated FeRh moments are represented by gray and white arrows, respectively. The springs illustrate the possible exchange coupling between them. c Unit vectors of uniaxial magnetic anisotropy for the single SM layer (black dots) and fourfold symmetrical anisotropy for the AF (red dots) and FM (blue dots) HM layers in the Monte Carlo simulation. d Calculated coercivity as a function of the field orientation for the single SM layer (black dots) and the SM/AF (red dots) and SM/FM (blue dots) bilayers. The oscillatory curves are the sinusoidally fitting results. The simulation indicates that the interfacial exchange coupling may result in a fourfold magnetic anisotropy of CoFeB layers with easy magnetization axes collinear with the magnetocrystalline anisotropy of FeRh, which is predicted to be e along

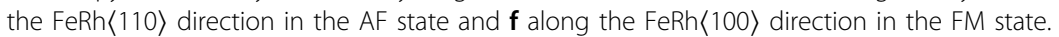

the coercivity indicates the extensive existence of rotatable FM FeRh moments coupling to the CoFeB moments across the interface. However, unlike other conventional EB systems, the negligible $\mathrm{EB}$ of $\mathrm{CoFeB} / \mathrm{FeRh}$ suggests almost no uncompensated pinned AF FeRh moments located at the interface, as schematically shown in Fig. 5a. After the occurrence of the AF-FM phase transition, all of the FeRh moments are FM and become rotatable (Fig. 5b). Thus, the EB disappears, but the interfacial exchange spring coupling between $\mathrm{CoFeB}$ and FeRh still exists.
A Monte Carlo simulation is employed to understand the effect of interfacial exchange coupling on forming the fourfold magnetic anisotropy in an amorphous FM layer. A SM (1 monolayer)/hard magnet (HM, 1 monolayer) bilayer structure with a lateral size of $100 \times$ 100 is established, where spins experience individual anisotropies and have four nearest neighbors in the same layer and four in the other layer to enhance interlayer (interfacial) and intralayer couplings. Under a magnetic field $H$, the Hamiltonian of the bilayer can 
be written as

$$
\begin{aligned}
\mathrm{H}= & -\sum_{i \in S M} K_{\mathrm{SM}}\left(S_{i} \cdot \widehat{e}_{i}^{S M}\right)^{2}-\sum_{<i j \in \mathrm{SM}>} J_{\mathrm{SM}} S_{i} \cdot S_{j} \\
& \left.-\sum_{i \in \mathrm{HM}} K_{\mathrm{HM}} \mid\left(S_{i}^{x}\right)^{4}+\left(S_{i}^{y}\right)^{4}\right\rfloor-\sum_{<i \in \mathrm{HM}>} J_{\mathrm{HM}} S_{i} \cdot S_{j} \\
& -\sum_{i} H\left(S_{i} \cdot \hat{e}_{i}^{\mathrm{H}}\right)-\sum_{<i j \in \mathrm{SM}, j \in \mathrm{HM}>} J_{\mathrm{IF}} S_{i} \cdot S_{j},
\end{aligned}
$$

where $S_{i}$ is the unit vector of spin $i$. The SM uniaxial and HM in-plane fourfold symmetrical magnetic anisotropies are considered, and the exchange interactions only occur between the nearest-neighbor spin pairs. In the simulation, the SM exchange constant $J_{\mathrm{SM}}=1.0$ is set, and other parameters are reduced by it to be dimensionless. The magnetic anisotropy constant is determined by the experimentally obtained coercivity $H_{c}$ based on a meanfield approximation where the coercivity is proportional to the magnetic anisotropy constant when spins are noninteracting. Thus, to obtain correct orders of magnitude of $H_{c}$ in the simulation, the magnetic anisotropy constants are set to $K_{\mathrm{SM}}=0.0375 J_{\mathrm{SM}}$ for the SM layer, $K_{\mathrm{HM}}=K_{\mathrm{AF}}=1.0 J_{\mathrm{SM}}$ for the HM layer in the AF state, and $K_{\mathrm{HM}}=K_{\mathrm{FM}}=-0.2 J_{\mathrm{SM}}$ for the HM layer in the FM state, where the sign of $K_{\mathrm{HM}}$ adjusts the easy axes oriented along the $x$ - and $y$-axes or along the middle angle lines between the $x$ - and $y$-axes. The HM exchange constants $J_{\mathrm{HM}}= \pm 0.1 J_{\mathrm{SM}}$ are used for the $\mathrm{FM}$ and AF states, respectively. The SM/HM interfacial exchange constant is $J_{\mathrm{IF}}=1.0 J_{\mathrm{SM}}$. The unit vectors of the SM easy axis and magnetic field are always set in the same direction due to the soft feature of the $\mathrm{SM}$, i.e., $\hat{e}_{i}^{\mathrm{SM}}=\hat{e}_{i}^{\mathrm{H}}=\left(90^{\circ}, \phi\right)$, where $\phi$ is an azimuthal angle varying from $0^{\circ}$ to $360^{\circ}$. Figure $5 \mathrm{c}$ gives the calculated unit vectors of the uniaxial magnetic anisotropy for the single SM layer and fourfold symmetrical anisotropy for the AF and FM HM layers in the Monte Carlo simulation.

Figure $5 \mathrm{~d}$ presents the obtained angular dependence of the coercivity for the single SM layer and the SM/AF and SM/FM bilayers. The coercivity of a single SM layer is roughly equal to 0.08 , two times larger than $K_{\mathrm{SM}}$ and independent of $\phi$, which is also consistent with the meanfield calculation result. When the SM layer is exchange coupled to an in-plane fourfold anisotropic AF layer, the coercivity sinusoidally oscillates between 0.5 and 1.0 with a periodicity of $90^{\circ}$. This periodicity indicates that the fourfold magnetic anisotropy of the AF layer is imprinted into the coercive behaviors of the amorphous SM layer. The coercivity of the SM/AF bilayer is an order of magnitude larger than that of the single SM layer, which is attributed to the rotation of the AF moment exchange coupled with the FM moment exchange in the SM layer. In contrast, when the amorphous SM layer is coupled to an in-plane fourfold anisotropic hard FM layer, the coercivity remains sinusoidally oscillatory with a periodicity of $90^{\circ}$ and displays a phase shift of $45^{\circ}$ compared to the SM/AF bilayer. The coercivity for the SM/FM bilayer at the equilibrium position reduces to one fourth of that for the SM/AF bilayer. The simulation results of both the decrease in the coercivity and the $45^{\circ}$ shift of the phase are consistent with the experimental observations. However, the simulations cannot give multistep magnetic switching behaviors; this is because the magnetization reversal is experimentally governed by DW nucleation and propagation. During the reversal process, the metastable states of the magnetization along the orthogonal easy axes can be detected, leading to the occurrence of multistep magnetic switching processes. However, in the simulation, a neat model of the SM/AF bilayer is used with an insufficient difference in the anisotropy strength between the $\mathrm{SM}$ and AF moments, full interfacial coupling and free of nonmagnetic defects. Meanwhile, at a finite temperature, taking into account thermal fluctuations, the fourfold symmetric anisotropy in the AF layer is further weakened. Consequently, only the symmetry and the variation in the coercivity are obtained. The same is true for the SM/FM bilayer.

The MAE of both AF and FM FeRh are calculated by the first-principle calculation considering the spin-orbit coupling. The MAE differences $\left(\mathrm{MAE}_{\langle 100\rangle-\langle 110\rangle}\right)$ are obtained by comparing the total energy of the magnetic moments oriented along the $\mathrm{FeRh}\langle 100\rangle$ and $\mathrm{FeRh}\langle 110\rangle$ directions. Structural relaxation is first performed until the force is smaller than $10^{-4} \mathrm{eV} / \AA$ to determine the ground state for FeRh in each geometry. The optimized lattice constants of the $\mathrm{AF}$ and $\mathrm{FM}$ cubic phases are obtained as 2.988 and $3.005 \AA$, respectively. The $\mathrm{MAE}_{\langle 100\rangle-\langle 110\rangle}$ are $1.540 \times 10^{3}$ and $-4.429 \times 10^{4} \mathrm{~J} / \mathrm{m}^{3}$ for $\mathrm{AF}$ and FM FeRh, respectively. This result indicates that the in-plane easy axes are along the $\mathrm{FeRh}\langle 110\rangle$ and FeRh $\langle 100\rangle$ directions for AF and FM FeRh, as shown in Fig. 5e, $f$, respectively. The calculated easy axes of FM FeRh are confirmed by the experimental observations shown in the inset of Fig. 1a. Moreover, the predicted easy axes of FeRh are collinear with the experimentally observed fourfold magnetic anisotropy of $\mathrm{CoFeB}$ regardless of $\mathrm{FeRh}$ in the AF or FM states. In addition, the impact of the epitaxial growth strain induced by the $\mathrm{MgO}(001)$ substrate on the magnetic ground state is investigated as well. A tetragonal distortion of $\mathrm{FeRh}$ lattices is considered to match the lattice constant of $\mathrm{MgO}(4.216 \AA)$, but the volume remains unchanged. The corresponding $c / a$ ratios are 1.006 and 1.024 for the AF and FM FeRh layers, respectively. The $\operatorname{MAE}_{\langle 100\rangle-\langle 110\rangle}$ become $2.045 \times 10^{4}$ and $-3.225 \times 10^{3} \mathrm{~J} / \mathrm{m}^{3}$ for AF and FM FeRh, respectively. This finding indicates that the magnetocrystalline anisotropy of $\mathrm{AF} F \mathrm{Fh}$ is approximately 6.3 times stronger than that of 
FM FeRh. In the experiment, the induced fourfold magnetic anisotropy in the CoFeB layers is found to decrease by a factor of 5.7 after the occurrence of the AF-FM phase transition of FeRh (Fig. 4c). The good agreement between the calculated and experimental results suggests that both the orientation and the strength of the fourfold magnetic anisotropy of the CoFeB layers are determined by the magnetocrystalline anisotropy of the FeRh layers.

Combining the Monte Carlo simulation and the firstprinciples calculation, we may understand the origin of the imprinted fourfold magnetic anisotropy in $\mathrm{CoFeB} /$ $\mathrm{FeRh}$ heterostructures. In the AF state of FeRh at room temperature, both the rotatable and pinned FeRh moments are aligned along the easy axes of the $\mathrm{FeRh}\langle 110\rangle$ directions due to the magnetocrystalline anisotropy. Consequently, the exchange coupling between the $\mathrm{CoFeB}$ moments and the rotatable FeRh moments across the interface produces an induced fourfold magnetic anisotropy along the $\mathrm{FeRh}\langle 110\rangle$ axes in the amorphous $\mathrm{CoFeB}$ layer, as schematically shown in Fig. 5e. The exchange coupling between the rotatable and pinned FeRh moments at the AF states may enhance the strength of the fourfold magnetic anisotropy. After the occurrence of the FeRh phase transition at an elevated temperature, the fourfold magnetic anisotropy continues to exist because of the similar exchange coupling between $\mathrm{CoFeB}$ and FeRh. However, the easy axes for FeRh in the FM state become along the $\mathrm{FeRh}\langle 100\rangle$ axes; thus, the induced fourfold magnetic anisotropy in the $\mathrm{CoFeB}$ layer changes to along the FeRh $\langle 100\rangle$ axes (Fig. 5f). Due to the reduced magnetocrystalline anisotropy of FM FeRh, the fourfold magnetic anisotropy of $\mathrm{CoFeB}$ becomes much weaker.

\section{Conclusions}

In summary, we fabricated heterostructures with an amorphous FM CoFeB layer on the top of an epitaxial metamagnetic $\mathrm{FeRh}$ layer on $\mathrm{MgO}(001)$ substrates. The MOKE and FMR measurements indicate that the amorphous $\mathrm{CoFeB}$ layer displays an in-plane fourfold magnetic anisotropy, which is usually observed in single crystal films. The easy axes of the CoFeB layer are along the $\mathrm{FeRh}\langle 110\rangle$ and $\mathrm{FeRh}\langle 100\rangle$ directions before and after the FeRh AF to FM transition, respectively. A first-principles calculation suggests that the fourfold magnetic anisotropy of the CoFeB layer is collinear with the magnetocrystalline anisotropy of the FeRh layer regardless of the magnetic states of FeRh. The inversely proportional dependence on the $\mathrm{CoFeB}$ thickness and the Monte Carlo simulation indicate that the imprinted fourfold magnetic anisotropy results from the interfacial exchange coupling between the $\mathrm{CoFeB}$ and FeRh moments. This phenomenon of imprinting the magnetocrystalline anisotropy of AF materials to the easily detected FM materials may be applied to probe the magnetic structures of $\mathrm{AF}$ materials without using synchrotron methods.

\section{Acknowledgements}

This work was financially supported by the National Natural Science Foundation of China (11874150, 11674336, 51871233, 51931011, 11774045, and 51525103), the Youth Innovation Promotion Association of the Chinese Academy of Sciences (2019299 and 2016270), Ningbo Natural Science Foundation (2019A610054), the Ningbo Science and Technology Bureau (2018B10060), the Ningbo Science and Technology Innovation Team (2015B11001), the Natural Science Foundation of Liaoning Science and Technology Department (20180510008), and the Fundamental Research Funds for the Central Universities (N180506001 and N182410008-1). A portion of this work was performed at the Steady High Magnetic Field Facilities, High Magnetic Field Laboratory, Chinese Academy of Sciences.

\section{Author details}

'Key Laboratory of Magnetic Materials and Devices, Ningbo Institute of Material Technology and Engineering, Chinese Academy of Sciences, Ningbo 315201, People's Republic of China. ${ }^{2}$ Key Laboratory of Polar Materials and Devices (MOE) and State Key Laboratory of Precision Spectroscopy, School of Physics and Electronic Science, East China Normal University, Shanghai 200241, People's Republic of China. ${ }^{3}$ Department of Physics, College of Sciences, Northeastern University, Shenyang 110819, People's Republic of China. ${ }^{4}$ State Key Laboratory of Rolling and Automation, Department of Physics, College of Sciences, Northeastern University, Shenyang 110819, People's Republic of China. ${ }^{5}$ High Magnetic Field Laboratory, Chinese Academy of Sciences, Hefei 230026, People's Republic of China

\section{Conflict of interest}

The authors declare that they have no conflict of interest.

\section{Publisher's note}

Springer Nature remains neutral with regard to jurisdictional claims in published maps and institutional affiliations.

Supplementary information is available for this paper at https://doi.org/ 10.1038/541427-020-00248-x.

Received: 28 February 2020 Revised: 12 August 2020 Accepted: 21 August 2020.

Published online: 16 October 2020

\section{References}

1. Johnson, M. T., Bloemen, P. J. H., den Broeder, F. J. A. \& de Vries, J. J. Magnetic anisotropy in metallic multilayers. Rep. Prog. Phys. 59, 1409 (1996).

2. van Vleck, J. H. On the anisotropy of cubic ferromagnetic crystals. Phys. Rev. 52, 1178-1198 (1937).

3. Wu, R. \& Freeman, A. J. Spin-orbit induced magnetic phenomena in bulk metals and their surfaces and interfaces. J. Magn. Magn. Mater. 200, 498-514 (1999).

4. Zhan, Q. F., Van Haesendonck, C., Vandezande, S. \& Temst, K. Surface morphology and magnetic anisotropy of Fe/MgO (001) films deposited at oblique incidence. Appl. Phys. Lett. 94, 042504 (2009).

5. Zhang, Y. et al. Magnetization reversal in epitaxial exchange-biased IrMn/FeGa bilayers with anisotropy geometries controlled by oblique deposition. Phys. Rev. B 91, 174411 (2015)

6. Stöhr, J. et al. Images of the antiferromagnetic structure of a NiO(100) surface by means of X-ray magnetic linear dichroism spectromicroscopy. Phys. Rev. Lett. 83, 1862-1865 (1999).

7. Donahue, M. J. \& Porter, D. G. Analysis of switching in uniformly magnetized bodies. IEEE Trans. Magn. 38, 2468-2470 (2002).

8. Meiklejohn, W. H. \& Bean, C. P. New magnetic anisotropy. Phys. Rev. 102, 1413-1414 (1956).

9. Chappert, C., Fert, A. \& Van Dau, F. N. The emergence of spin electronics in data storage. Nat. Mater. 6, 813-823 (2007).

10. Wolf, S. A. et al. Spintronics: a spin-based electronics vision for the future Science 294, 1488-1495 (2001).

11. Phuoc, N. N., Xu, F. \& Ong, C. K. Ultrawideband microwave noise filter: hybrid antiferromagnet/ferromagnet exchange-coupled multilayers. Appl. Phys. Lett. 94, 092505 (2009) 
12. Chu, Y. H. et al. Electric-field control of local ferromagnetism using a magnetoelectric multiferroic. Nat. Mater. 7, 478-482 (2008).

13. Parkin, S. S. et al. Giant tunnelling magnetoresistance at room temperature with MgO (100) tunnel barriers. Nat. Mater. 3, 862-867 (2004).

14. Ikeda, S. et al. H. A perpendicular-anisotropy CoFeB-MgO magnetic tunnel junction. Nat. Mater. 9, 721-724 (2010).

15. Kouvel, J. S. \& Hartelius, C. C. Anomalous magnetic moments and transformations in the ordered alloy FeRh. J. Appl. Phys. 33, 1343-1344 (1962).

16. Zakharov, A. I., Kadomtseva, A. M., Levitin, R. Z. \& Ponyatovskii, E. G. Magnetic and magnetoelastic properties of a metamagnetic iron-rhodium alloy. Sov. Phys. JETP 19, 1348-1353 (1964).

17. Annaorazov, M. P., Nikitin, S. A., Tyurin, A. L., Asatryan, K. A. \& Dovletov, A. K Anomalously high entropy change in FeRh alloy. J. Appl. Phys. 79, 1689-1695 (1996).

18. van Driel, J., Coehoorn, R., Strijkers, G. J., Bruck, E. \& de Boer, F. R. Compositional dependence of the giant magnetoresistance in $\mathrm{Fe}_{x} \mathrm{Rh}_{1-x}$ thin films. J. Appl. Phys. 85, 1026-1036 (1999).

19. Lee, Y. et al. Large resistivity modulation in mixed-phase metallic systems. Nat. Commun. 6, 5959 (2015)

20. Baldasseroni, $\mathbf{C}$. et al. Temperature-driven nucleation of ferromagnetic domains in FeRh thin films. Appl. Phys. Lett. 100, 262401 (2012).

21. Suzuki, I., Hamasaki, Y., Itoh, M. \& Taniyama, T. Controllable exchange bias in Fe/metamagnetic FeRh bilayers. Appl. Phys. Lett. 105, 172401 (2014).

22. Nam, N. T., Lu, W. \& Suzuki, T. Exchange bias of ferromagnetic/antiferromagnetic in FePt/FeRh bilayers. J. Appl. Phys. 105, 070708 (2009).

23. Thiele, J.-U., Maat, S. \& Fullerton, E. E. FeRh/FePt exchange spring films for thermally assisted magnetic recording media. Appl. Phys. Lett. 82, 2859-2861 (2003).

24. Heim, B., Rønnow, T. F., Isakov, S. V. \& Troyer, M. Quantum versus classical annealing of Ising spin glasses. Science 348, 215 (2015).

25. Hu, Y. et al. Magnetocaloric effect in cubically anisotropic magnets. Appl. Phys. Lett. 114, 023903 (2019).

26. $\mathrm{Hu}, \mathrm{Y}$. Prediction of reentering and switching ferromagnet/antiferromagnet exchange bias by antiferromagnetic proximity effect. Nanotechnology $\mathbf{3 0}$, 025708 (2019).

27. Kresse, G. \& Furthmuller, J. Efficiency of ab-initio total energy calculations for metals and semiconductors using a plane-wave basis set. Comput. Mater. Sci. 6, 15 (1996).

28. Blochl, P. E. Projector augmented-wave method. Phys. Rev. B 50, 17953 (1994)

29. Perdew, J. P., Burke, K. \& Ernzerhof, M. Generalized gradient approximation made simple. Phys. Rev. Lett. 77, 3865 (1996)
30. Kresse, G. \& Joubert, D. From ultrasoft pseudopotentials to the projector augmented-wave method. Phys. Rev. B 59, 1758 (1999).

31. Kim, C. G., Rheem, Y. W., Kim, C. O., Shalyguina, E. E. \& Ganshina, E. A. Magnetostatic properties of heterogeneous Co-based amorphous/crystalline phases. J. Magn. Magn. Mater. 262, 412-419 (2003).

32. Yuasa, S., Nývlt, M., Katayama, T. \& Suzuki, Y. Exchange coupling of NiFe/FeRh-lr thin films. J. Appl. Phys. 83, 6813-6815 (1998).

33. Arenholz, E. \& Liu, K. Angular dependence of the magnetization reversal in exchange-biased Fe/MnF 2 . Appl. Phys. Lett. 87, 132501 (2005).

34. Blomqvist, P., Krishnan, K. M. \& Ohldag, H. Direct imaging of asymmetric magnetization reversal in exchange-biased Fe/MnPd bilayers by X-ray photoemission electron microscopy. Phys. Rev. Lett. 94 107203 (2005)

35. Zhan, Q. F., Zhang, W. \& Krishnan, K. M. Antiferromagnetic layer thickness dependence of the magnetization reversal in the epitaxial MnPd/Fe exchange bias system. Phys. Rev. B 83, 094404 (2011).

36. Cowburn, R. P., Gray, S. J., Ferré, J., Bland, J. A. C. \& Miltat, J. Magnetic switching and in-plane uniaxial anisotropy in ultrathin Ag/Fe/Ag (100) epitaxial films. J. Appl. Phys. 78, 7210-7219 (1995).

37. Zhan, Q. F., Vandezande, S., Van Haesendonck, C. \& Temst, K. Magnetic anisotropy and reversal in epitaxial Fe/MgO (001) films. Phys. Rev. B 80, 094416 (2009).

38. Daboo, C. et al. Anisotropy and orientational dependence of magnetization reversal processes in epitaxial ferromagnetic thin films. Phys. Rev. B 51, 15964 (1995).

39. Heidarian, A. et al. Ferromagnetic resonance of MBE-grown FeRh thin films through the metamagnetic phase transition. Phys. Status Solidi B 254, 1700145 (2017).

40. Huang, Z. C. et al. The interface effect of the magnetic anisotropy in ultrathin epitaxial $\mathrm{Fe}_{3} \mathrm{O}_{4}$ film. Appl. Phys. Lett. 92, 113105 (2008).

41. Manna, P. K. \& Yusuf, S. M. Two interface effects: exchange bias and magnetic proximity. Phys. Rep. 535, 61 (2014).

42. Camarero, J. et al. Magnetization reversal, asymmetry, and role of uncompensated spins in perpendicular exchange coupled systems. Appl. Phys. Lett. 89, 232507 (2006)

43. Brück, S., Schütz, G., Goering, E., Ji, X. S. \& Krishnan, K. M. Uncompensated moments in the MnPd/Fe exchange bias system. Phys. Rev. Lett. 101, 126402 (2008).

44. Wu, J. et al. Direct measurement of rotatable and frozen $\mathrm{CoO}$ spins in exchange bias system of CoO/Fe/Ag(001). Phys. Rev. Lett. 104, 217204 (2010) 